\title{
Terapia de substituição renal em pacientes acometidos pela Covid-19 em uma Unidade de Terapia Intensiva no interior de Rondônia
}

\author{
Renal replacement therapy in patients affected by Covid-19 in an Intensive Care Unit in the \\ countryside of Rondônia \\ Terapia de reemplazo renal en pacientes afectados por Covid-19 en una Unidad de Cuidados \\ Intensivos en el campo de Rondônia
}

Recebido: 25/11/2021 | Revisado: 03/12/2021 | Aceito: 19/12/2021 | Publicado: 24/12/2021

\author{
Sara Ferreira Sottocornola \\ ORCID: https://orcid.org/0000-0003-4226-6475 \\ Hospital Regional de Cacoal, Brasil \\ E-mail: sarasotts@gmail.com \\ Rittiela Rocha da Silva \\ ORCID: https://orcid.org/0000-0003-0409-1360 \\ Hospital Regional de Cacoal, Brasil \\ E-mail: rittielarocha@ hotmail.com \\ Shirley Junqueira Barbosa \\ ORCID: https://orcid.org/0000-0003-4609-0542 \\ Hospital Regional de Cacoal, Brasil \\ E-mail: shirley.jjunqueira@gmail.com \\ Amanda Gabrielle Silva Queiroz \\ ORCID: https://orcid.org/0000-0002-8161-5124 \\ Hospital Regional de Cacoal, Brasil \\ E-mail: gabrielle.pb@hotmail.com \\ Rafael Zondonadi de Souza \\ ORCID: https://orcid.org/0000-0002-8132-2313 \\ Hospital Regional de Cacoal, Brasil \\ E-mail: rafaelzondonadi@gmail.com \\ Érico Minoru Narahashi Bulgarelli \\ ORCID: https://orcid.org/0000-0001-8667-9875 \\ Centro de Pesquisa em Medicina Tropical, Brasil. \\ E-mail: minouru@hotmail.com
}

\begin{abstract}
Resumo
A pesquisa foi realizada a partir da análise de prontuários de pacientes que adquiriram Lesão Renal Aguda (LRA) e necessitaram da Terapia de Substituição Renal (TSR) nas internações em Unidades de Terapia Intensiva Covid do Hospital Regional de Cacoal (HRC) no Interior de Rondônia. É um estudo descritivo, transversal, retrospectivo e de abordagem quantitativa. Após aplicar os critérios de exclusão, ficaram aptos para a análise 188 prontuários no período de junho de 2020 a junho de 2021. Dos 188 pacientes, 108 pessoas eram do sexo masculino (57\%), com média de idade de 62,44, mínima de 23 e máxima de 94 anos. Diabetes, hipertensão e obesidade foram as doenças pré-existentes mais prevalentes no estudo com $84 \%$, e $6 \%$ dos pacientes desenvolveram LRA sem ter nenhuma comorbidade declarada. Observa-se nesse estudo um índice elevado de pacientes que evoluíram para a hemodiálise com um desfecho alto de mortalidade com os exames laboratoriais alterados, totalizando $93 \%$ dos óbitos e $7 \%$ de altas. A LRA em pacientes internados com a Covid-19 está entrelaçada a um mau prognóstico com um grande índice de mortalidade, que o estado hiper inflamatório afeta as células renais, ocasionando a necessidade da terapia de substituição renal.
\end{abstract}

Palavras-chave: Covid-19; Unidades de Terapia Intensiva; Injúria Renal Aguda.

\begin{abstract}
The research was carried out from the analysis of medical records of patients who acquired Acute Kidney Injury (AKI) and needed Kidney Replacement Therapy (RRT) in admissions to Covid Intensive Care Units of the Hospital Regional de Cacoal (HRC) in the countryside of Rondônia. It is a descriptive, cross-sectional, retrospective study with a quantitative approach. After applying the exclusion criteria, 188 medical records were able to be analyzed from June 2020 to June 2021. Of the 188 patients, 108 were male (57\%), with a mean age of 62.44, minimum age 23 and maximum 94 years. Diabetes, hypertension and obesity were the most prevalent pre-existing diseases in the study with $84 \%$, and $6 \%$ of patients developed AKI without having any declared comorbidity. In this study, there is a high rate of patients who evolved to hemodialysis with a high mortality outcome with altered laboratory tests, totaling $93 \%$ of deaths and
\end{abstract}


$7 \%$ of discharges. AKI in patients hospitalized with Covid-19 is intertwined with a poor prognosis with a high mortality rate, that the hyperinflammatory state affects the renal cells, causing the need for renal replacement therapy.

Keywords: Covid-19; Intensive Care Units; Acute Kidney Injury.

\section{Resumen}

La investigación se llevó a cabo a partir del análisis de historias clínicas de pacientes que adquirieron Lesión Renal Aguda (LRA) y necesitaron Terapia Reemplazo de Riñón (TRS) en los ingresos a las Unidades de Cuidados Intensivos Covid del Hospital Regional de Cacoal (HRC) en el campo de Rondônia. Se trata de un estudio descriptivo, transversal, retrospectivo con enfoque cuantitativo. Tras aplicar los criterios de exclusión, se pudieron analizar 188 historias clínicas desde junio de 2020 a junio de 2021. De los 188 pacientes, 108 eran varones (57\%), con una edad media de 62,44, edad mínima 23 y máxima 94 años. La diabetes, la hipertensión y la obesidad fueron las enfermedades preexistentes más prevalentes en el estudio con un $84 \%$ y el $6 \%$ de los pacientes desarrollaron LRA sin tener ninguna comorbilidad declarada. En este estudio, existe una alta tasa de pacientes que evolucionaron a hemodiálisis con un resultado de alta mortalidad con pruebas de laboratorio alteradas, totalizando el 93\% de las muertes y el 7\% de los egresos. La LRA en pacientes hospitalizados con Covid-19 se entrelaza con un mal pronóstico con una alta tasa de mortalidad, que el estado hiperinflamatorio afecta las células renales, provocando la necesidad de terapia de reemplazo renal.

Palabras clave: Covid-19; Unidades de Cuidados Intensivos; Lesión Renal Aguda.

\section{Introdução}

A Lesão Renal Aguda (LRA) em pacientes Covid-19 é multifatorial nas internações, sendo evidenciada como uma das complicações mais comuns acometendo tanto pacientes portadores de uma doença renal pré-existente quanto quem não possuía nenhuma comorbidade. Estudo in vitro de células epiteliais tubulares renais demonstrou que a Síndrome Respiratória Aguda Grave proveniente da infecção da Covid-19, estabeleceu em células renais proximais uma infecção persistente e produtiva relacionado com parte da expressão a enzima de conversão da angiotensina 2 (ACE2), porém existe outra vertente que mostra o excesso de medicamentos em tratamento dos pacientes graves em ambientes hospitalares, a intoxicação medicamentosa pode acometer o quadro de insuficiência renal, pois a eliminação das drogas depende totalmente da função renal, uma vez que a reposição de fluidos é deficiente podendo levar o rim a ter uma hipoperfusão (Nunes, 2020).

A hipótese inicial que abrange a gravidade da Covid-19 é estabelecida por uma resposta inflamatória intensa que ocorre no nosso organismo quando entramos em contato com o vírus. Relaciona-se que a insuficiência respiratória está interligada à ACE2 e que pode ser encontrada nas células epiteliais tubulares renais (Hidalgo-Blanco, 2020).

A terapia de substituição renal (TSR) que tem como principal forma a hemodiálise, pode ser utilizada para fornecer um suporte renal em pacientes críticos com instabilidade hemodinâmica, sendo a lesão renal aguda uma complicação frequente nos pacientes críticos e associa-se a elevados índices de mortalidade dentro das Unidades de Terapia Intensiva (UTI) (Tandukar S. 2019; Alvarez, 2019).

O desenvolvimento da LRA em pacientes com Covid-19 durante as internações se implica em falha sistêmica, iniciandose pela insuficiência respiratória aguda, indicando a falha do sistema respiratório, precisando assim do tubo orotraqueal e da ventilação mecânica invasiva. A evolução da doença na forma mais severa causa uma hiper inflamação e pode levar o paciente a ter uma injúria renal, pela ligação do vírus às células renais causando uma forma grave de disfunção renal. Em geral essa associação correlaciona-se com uma piora do prognóstico, elevando a mortalidade. A LRA é uma realidade de alguns pacientes durante as internações, tendo como indicação o serviço nefrológico da terapia de substituição renal (Kunutsor, 2020; Tandukar S. 2019; Suassuna, et al., 2020).

\subsection{Lesão Renal Aguda}

A função dos rins é realizar a homeostase, equilibrando o ambiente interno e mantendo com segurança cerca de 100 trilhões de células do corpo humano. Exercendo múltiplas funções como filtrar e reabsorver. A fisiologia renal abrange informações importantes desde a filtração até a formação da diurese, qualquer alteração interna e urinária pode causar uma 
disfunção, podendo causar danos celulares. Cerca de 1.200 a $1.500 \mathrm{ml}$ de sangue chegam aos rins e é filtrado pelos glomérulos, túbulo coletor proximal e distal, alça de Henle e o ducto coletor, são responsáveis de reabsorver e secretar os íons, os hormônios que regulam o sistema são a renina-angiotensina-aldosterona e o hormônio antidiurético (Younes, 2021; Sodré, 2021).

A LRA é definida como um declínio agudo da função renal em horas ou dias. Significa principalmente uma diminuição na taxa de filtração glomerular e /ou produção de urina. No entanto, também existem interferências no controle do equilíbrio de água, eletrólito e ácido alcalino. O que ocasiona uma retenção de escórias metabólicas, ureia e creatinina, e é definida quando há uma elevação do valor da creatinina sérica em um período de 48 horas. Como característica tem a redução do volume urinário, acúmulo de líquido na forma de edema, disfunção da diluição urinária, perda da capacidade de regular o equilíbrio ácido básico e disfunção nos níveis de potássio, sódio, magnésio, cálcio e fósforo (Martins, 2014; Sociedade Brasileira de Nefrologia, 2014).

Sendo assim a LRA pode ser dividida em:

- $\quad$ LRA Pré Renal: Quando ocorre uma hipoperfusão renal, mas a integridade do parênquima renal é preservada;

LRA Renal: Ocorre quando há dano tissular;

LRA Pós Renal: Quando ocorre obstrução entre o meato uretral externo e o trígono vesical (Martins, 2014).

\subsection{Covid-19 e Lesão Renal Aguda}

No final de dezembro de 2019, a pandemia Covid-19 surgiu em Wuhan, província de Hubei, China, quando 44 casos de pneumonia de etiologia desconhecida foram notificados à Organização Mundial de Saúde (OMS), dos quais 11 eram pacientes gravemente enfermos. No dia 30 de janeiro de 2020 foi declarada uma Emergência de Saúde Pública de Importância Internacional (ESPIN) com um importante grau de propagação. Em 25 de fevereiro de 2020, 81.109 casos confirmados foram documentados em todo o mundo, a OMS declarou uma emergência de saúde pública de interesse internacional. A pandemia causada pelo vírus Covid-19 representou um desafio para o sistema de saúde pública com um índice de morbidade e mortalidade alarmantes, considerado mais expressivo do que a gripe espanhola em 1918. Afetando o sistema respiratório, geralmente se manifestando como uma síndrome do desconforto respiratório agudo (SDRA) e pneumonia grave, a maioria dos pacientes é assintomática ou tem sintomas leves (Kunutsor, 2020; Holshue et al., 2020; Guan, 2020).

Os principais sintomas da patologia são febre, tosse, dispneia, mialgia, anosmia e ageusia. O público idoso, homens e com comorbidades preexistentes, em especial a hipertensão, diabetes, doença hepática e renal crônica estão mais suscetíveis a se infectar com a forma grave da doença, podendo causar falência dos órgãos e eventualmente levar ao óbito. Nos casos graves, a Covid-19, afeta outros órgãos extrapulmonares, o comprometimento renal é um deles, o distúrbio hidroeletrolítico é evidenciado com mais frequência durante as internações. Sabe-se que o vírus interliga as células pulmonares pela ACE2, atingindo assim vários outros órgãos. O rim tem como expressão maior em várias células (mesangiais, podócitos, epitélio parietal da cápsula de Bowman e os dutos coletores) podendo causar essa disfunção renal e uma cascata de citocinas devido a inflamação sistêmica, embora ainda não esteja tão claro essa interligação do vírus com a LRA (Queiroz et al., 2020; Scholz, 2020; Sosa, 2020; Guan, 2020).

A LRA quando acomete os pacientes hospitalizados oferece uma condição de 0,5-25\% de mau prognóstico. A pneumonia ocasionada pela Covid-19 pode ter acometimento renal, em um estudo com 251333 pacientes $(75,4 \%)$ tinham doença renal, esse envolvimento está sendo conjugado com a gravidade da infecção pelo vírus, aumentando dessa forma o índice de mortalidade (Wei-jie Guan et al.,2020; Moitinho, 2020).

\section{Metodologia}

O estudo realizado a partir da análise de prontuários dos pacientes que adquiriram LRA e necessitaram de Terapia de Substituição Renal (TSR) nas internações em Unidades de Terapia Intensiva Covid (01, 03 e 04) do Hospital Regional de Cacoal 
(HRC), a hemodiálise convencional foi o modo escolhido de diálise. O HRC foi adaptado para o atendimento intensivo aos pacientes que atingiram a forma grave da Covid-19, tornando-se hospital de referência no interior do estado de Rondônia. Os critérios de inclusão são pacientes que adquiriram LRA durante a internação nas Unidades Covid 1,3 e 4 durante o período de junho de 2020 a junho de 2021 e pacientes maiores de 18 anos. Os critérios de exclusão são os que já possuíam histórico de Doença Renal Crônica com utilização da fístula arteriovenosa e população indígena. É um estudo descritivo, transversal, retrospectivo e de abordagem quantitativa. A amostra de dados foi obtida pela coleta realizada pelos autores desse presente estudo nos prontuários, os dados coletados foram analisados em forma de planilha no programa Excel: Número de prontuário, unidade hospitalar, sexo, idade, data de admissão, início da hemodiálise, diagnóstico principal, comorbidades, exames laboratoriais (potássio, sódio, creatinina, uréia) e o desfecho da internação.

É garantido que a presente pesquisa não ofereceu nenhum risco aos pacientes envolvidos, pois não houve procedimentos para a coleta de amostras biológicas ou outros que geram riscos diretos aos participantes por se tratar de estudo documental com a liberação a dispensa do Termo de Consentimento Livre e Esclarecido (TCLE) e aprovação pelo Comitê de Ética em Pesquisa da Faculdade de Ciências Biomédicas de Cacoal - FACIMED, CAAE: 46296921.2.0000.5298, Número do Parecer: 4.702.938.

\section{Resultados}

No período estipulado pelo estudo, 215 pacientes necessitaram da TSR e foram diagnosticados com a Covid-19. Após aplicar os critérios de exclusão, ficaram aptos para a análise 188 prontuários no período de junho de 2020 a junho de 2021. Dos 188 pacientes, 108 pessoas eram do sexo masculino (57\%), com média de idade de 62,44, mínima de 23 e máxima de 94 anos, desvio Padrão 14,46. A Unidade 3 teve o maior público de pacientes que realizaram hemodiálise 38\%, seguindo a ordem com a Unidade 1 com $36 \%$ e a Unidade 4 com $26 \%$.

Diabetes, hipertensão e obesidade foram às doenças pré-existentes mais prevalentes no estudo com $84 \%$, e $6 \%$ dos pacientes desenvolveram LRA sem ter nenhuma comorbidade declarada. (Tabela 1)

Os exames laboratoriais analisados anteriormente à hemodiálise foram a creatinina, potássio, sódio e a ureia. Os resultados mais alterados foram observados nos exames de Creatinina 187/99\%, média de 4.2 e o desvio padrão 2,843 e de Ureia 185/99\%, média 140,34, desvio padrão 59,126. (Tabela 2)

O tempo mínimo de internação da UTI para o início da diálise foi de 0 a 46 dias, ou seja, pacientes internaram com urgência dialítica, tempo médio de 6,45 dias e o desvio padrão de 6,868. Do início da hemodiálise para o desfecho da internação foram de 0-79 dias, média de 9,1 dias, desvio padrão 12,356, demonstrando que pacientes recém-internados que inseriram o cateter de diálise tiveram a conclusão da internação, totalizando 93\% dos óbitos e 7\% de altas. (Tabela 3)

Tabela 1 - Características gerais dos pacientes internados que iniciaram hemodiálise nas Unidades Terapia Intensiva de um hospital público da cidade de Cacoal, Rondônia, Brasil, 2021.

\begin{tabular}{ccc}
\hline & CARACTERÍSTICAS GERAIS & \\
Variável & $\boldsymbol{N}$ & $\%$ \\
Sexo Masculino & 108 & $57 \%$ \\
Sexo Feminino & 80 & $43 \%$ \\
Unidade 1 & 68 & $36 \%$ \\
Unidade 3 & 71 & $38 \%$ \\
Unidade 4 & 49 & $26 \%$ \\
Diabetes, hipertensão e obesidade & 157 & $84 \%$ \\
Doenças Pulmonares & 9 & $5 \%$ \\
Outras Comorbidades & 10 & $5 \%$ \\
Sem Comorbidades & 12 & $6 \%$ \\
\hline
\end{tabular}

Fonte: Dados da pesquisa (2021). 
Tabela 2 - Exames laboratoriais na admissão do setor de hemodiálise na cidade de Cacoal, Rondônia, Brasil, 2021.

\begin{tabular}{|c|c|c|c|c|c|c|}
\hline \multicolumn{7}{|c|}{ EXAMES LABORATORIAIS PRÉ HEMODIÁLISE } \\
\hline VARIÁVEL & $\mathrm{N}$ & $\%$ & MÍNIMO & MÁXIMO & MÉDIA & $\begin{array}{l}\text { DESVIO } \\
\text { PADRÃO }\end{array}$ \\
\hline \multicolumn{7}{|l|}{ CREATININA } \\
\hline Abaixo & - & - & - & - & - & - \\
\hline Normal & 1 & $1 \%$ & 1 & 1 & 1 & \\
\hline Acima & 187 & $99 \%$ & 1 & 27 & 4,2 & 2,843 \\
\hline Total & 188 & $100 \%$ & & & & \\
\hline \multicolumn{7}{|l|}{ POTÁSSIO } \\
\hline Abaixo & 3 & $2 \%$ & 5 & 6 & 5,27 & 0,252 \\
\hline Normal & 51 & $27 \%$ & 4 & 9 & 6,87 & 1,394 \\
\hline Acima & 134 & $71 \%$ & 2 & 12 & 6,34 & 1,545 \\
\hline Total & 188 & $100 \%$ & & & & \\
\hline \multicolumn{7}{|l|}{ SÓDIO } \\
\hline Abaixo & 55 & $29 \%$ & 110 & 176 & 139,95 & 10,616 \\
\hline Normal & 80 & $43 \%$ & 124 & 184 & 141,27 & 10,923 \\
\hline Acima & 53 & $28 \%$ & 126 & 188 & 141,5 & 10,143 \\
\hline Total & 188 & $100 \%$ & & & & \\
\hline \multicolumn{7}{|l|}{ UREIA } \\
\hline Abaixo & - & - & - & - & - & - \\
\hline Normal & 1 & $1 \%$ & 153 & 153 & 153 & - \\
\hline Acima & 185 & $99 \%$ & 8 & 438 & 140,34 & 59,126 \\
\hline Total & 186 & $100 \%$ & & & & \\
\hline
\end{tabular}

Fonte: Dados da pesquisa (2021).

Tabela 3 - Desfecho de internação dos pacientes em terapia de substituição renal na cidade de Cacoal, Rondônia, Brasil, 2021.

\begin{tabular}{ccccc}
\hline \multicolumn{5}{c}{ DESFECHO DE INTERNAÇÃO } \\
VARIÁVEL & UNIDADE 1 & UNIDADE 3 & UNIDADE 4 & TOTAL \\
ÓBITO & 61 & 67 & 46 & 174 \\
$\%$ & $32 \%$ & $36 \%$ & $25 \%$ & $93 \%$ \\
ALTA & 7 & 4 & 3 & 14 \\
$\%$ & $4 \%$ & $2 \%$ & $2 \%$ \\
\hline \multicolumn{5}{r}{ Fonte: Dados da pesquisa (2021). }
\end{tabular}

\section{Discussão}

A LRA nos pacientes diagnosticados com a Covid-19 apresenta-se de forma complexa e com diversos fatores. Observando que além das comorbidades prévias, a alteração hemodinâmica juntamente com a liberação de citocinas e a citotoxicidade do vírus com a ativação da angiotensina II que é receptor do vírus, podendo ser visto nas células renais como também nas células pulmonares. Pressupõe-se que o desenvolvimento de LRA nos pacientes é o resultado de um estado hiper inflamatório, ocasionando muitas vezes uma disfunção de múltiplos órgãos. Em uma amostra do estado do Rio de Janeiro no ano de 2020, 102 pacientes foram diagnosticados com LRA, no período de 17 de março a 26 de abril. A hipertensão e diabetes foram as doenças mais prevalentes encontradas com 53,9\% e 31,4\% da população geral, respectivamente (Costa, 2021; Pecly et al., 2021). O que vem a corroborar com essas variáveis que foram vistas neste estudo com no grupo diabetes, hipertensão e obesidade em $84 \%$ dos pacientes (157/188).

Um estudo comparativo da incidência da LRA entre três países em 2020: China, Itália e Estados Unidos demonstraram que a taxa de mortalidade foi elevada nos pacientes que possuíam comorbidades instaladas. A creatinina e a ureia na corrente sanguínea tendem a elevar-se com a deterioração do estado clínico e principalmente antes da morte, o nível médio dos exames na China acima do valor normal em pacientes críticos era de 4,3\%, (6/138). Nos EUA, o nível médio é de 1, 45\%, número consideravelmente maior que na China. Na Itália não apresentaram alterações registradas sobre a anormalidade desses exames. Os casos de LRA em pacientes críticos na China foram de 2,9\% (5/173). Nos EUA, os pacientes graves foram de 19,1\%, (4/21). 
22,6\% dos pacientes mortos com LRA na Itália são a segunda maior causa de morte após a SDRA (Chen, 2020). No Hospital Regional de Cacoal (HRC), onde foi realizada a pesquisa deste estudo, teve um desfecho elevado de mortalidade com $93 \%$ dos óbitos e 7\% de altas em um período de 1 ano.

Em uma pesquisa espanhola no Hospital Universitario Puerta de Hierro, no período de 25 de fevereiro a 24 de abril de 2020, com 1.603 pacientes internados. A idade média foi de 64 anos e 59,6\% dos pacientes eram do sexo masculino. Os dados obtidos neste estudo tiveram os dados similares, 108 pacientes eram do sexo masculino (57\%), com a idade média 62,44 anos. As principais comorbidades observadas na admissão foram hipertensão (35,7\%), obesidade (20,3\%), diabetes (15,2\%) e com tratamento imunossupressor prévio (2,9\%), 5,1\% pacientes necessitam de diálise, o que vai de encontro com o presente estudo que trouxe como grupo de comorbidades (hipertensão, diabetes e obesidade), e evoluíram para LRA com maior incidência (84\%). A mortalidade hospitalar foi maior em pacientes que tinham exame de creatinina elevado e que desenvolveram LRA durante a internação $(15,9 \%)$ em comparação àqueles que possuíam o exame nas taxas de normalidade (5,8\%) (Portolés et al., 2020).

Estudo retrospectivo chinês em 2020 no Hospital Tongji de Sino-French New City District, a média de idade foi de 56,3 anos e 54,7\% pacientes eram homens. A prevalência de hipertensão e diabetes foi de 32,2\% (107 de 333) e 22,9\% (76 de 333). A incidência de LRA em sua totalidade final foi de 4,7\% (22 de 467) pelos critérios KIDGO e 7,5\% (35 de 467) pelos critérios expandidos. Pacientes com prejuízo renal tiveram mortalidade mais elevada em comparação aos sem envolvimento renal. (Pei et al., 2020). Em 13 hospitais acadêmicos e comunitários na região metropolitana de Nova York no Hospital Northwell Health no período de $1^{\circ}$ de março de 2020 a 5 de abril de 2020, demonstrou nos seus resultados que 1993 de 5449 pacientes (36,6\%) desenvolveram LRA durante a hospitalização. O número de pacientes que necessitaram de terapia de suporte dialítico foi de 285 (5,2\% de todos os pacientes, $14,3 \%$ dos pacientes com LRA). Os preditores para LRA foram pacientes com idade avançada, sexo masculino, diabetes mellitus, hipertensão, doença cardiovascular (Hirsch et al., 2020). Segundo um estudo observacional de complicações renais em pacientes com Covid-19, o distúrbio eletrolítico mais comum foi a hipercalemia, durante internação hospitalar variando de 2 a 28 dias, com incidência de LRA a 11\% e necessidade de diálise 6,8\%, sendo a complicação renal mais frequente (kunutsor, 2020).

Estudos retrospectivos de uma revisão sistêmica e meta-análise em Abril de 2020, onde relataram riscos elevados de complicações graves nos pacientes infectados pela Covid-19 com certas doenças crônicas preexistentes, uma pesquisa envolvendo 59 pacientes, verificou-se que 32 de 51 indivíduos (63\%) apresentaram proteinúria. 19\% com o exame de creatinina e $27 \%$ de ureia elevado. Foi evidenciado que $29 \%$ dos pacientes apresentaram LRA (Wang, 2020).

A mortalidade hospitalar, segundo uma coorte prospectiva de 1.603 pacientes de um Hospital Universitário de Referência no centro europeu em 2020, foi maior em pessoas que tinham o exame de creatinina elevado (32,4\%), uma análise demonstrou que tinham mais óbitos os pacientes que eram admitidos com a creatinina (Portolés et al., 2020). O exame de creatinina neste estudo apresentou alteração em $99 \%$ dos pacientes (187/188), seguido da ureia com 185/188 pacientes.

\section{Considerações Finais}

Observa-se nesse estudo um índice elevado de pacientes que evoluíram para a hemodiálise convencional com um desfecho alto de mortalidade, os exames laboratoriais alterados evidenciaram uma condição que a função renal foi prejudicada. A lesão renal aguda em pacientes internados com a Covid-19 está relacionada a um mau prognóstico, onde o estado hiper inflamatório afeta as células renais, ocasionando a necessidade da terapia de substituição renal.

As informações contidas nesse estudo demonstraram a importância de uma equipe multiprofissional capacitada e com olhar clínico para que alterações hemodinâmicas sejam precocemente observadas, podendo assim agir o quanto antes na terapêutica adequada para cada paciente, podendo ajudar a melhorar o prognóstico. 


\section{Referências}

Alvarez, G., Chrusch, C., Hulme, T., \& Posadas-Calleja, J. G. (2019). Renal replacement therapy: a practical update. Traitement substitutif de l'insuffisance rénale: une mise à jour pratique. Canadian journal of anaesthesia = Journal canadien d'anesthesie, 66(5), 593-604. https://doi.org/10.1007/s12630-019-01306-

Chen, L., \& Guo, C. (2020). Focus on kidney disease among the coronavirus disease 2019 patients: A comparative perspective between China, Italy and the United States. International journal of clinical practice, 74(9), e13561. https://doi.org/10.1111/ijcp.13561

Costa, R. L., Sória, T. C., Salles, E. F., Gerecht, A. V., \& Corvisier, M. F. (2021). Acute kidney injury in patients with Covid-19 in a Brazilian ICU: incidence, predictors and in-hospital mortality. Braz. J. Nephrol. (J. Bras. Nefrol.) 2021;43(3):349-358. https://doi.org/10.1590/2175- 8239-JBN-2020-0144

Wei-jie Guan, P. D., Zheng-yi Ni, M. D., Yu Hu, M. D et al. (2020). Clinical Characteristics of Coronavirus Disease 2019 in China..The New England Journal of Medicine , 382 (18), 1708-1720. https://doi.org/10.1056/NEJMoa2002032

Pei, G., Zhang, Z., Peng, J., Liu, L., Zhang, C et al. (2020). Renal Involvement and Early Prognosis in Patients with Covid-19 Pneumonia. Journal of the American Society of Nephrology. 31(6),1157-1165. https://doi.org/10.1681/ASN.2020030276

Hidalgo-Blanco, M. A., Andreu-Periz, D., \& Moreno-Arroyo, M. C. (2020). Covid-19en el enfermo renal. Revisión breve. Enfermería Nefrológica, 23(2), 122131. https://dx.doi.org/10.37551/s2254-28842020013

Hirsch, J. S., Ng, J. H., Ross, D. W., Sharma, P., Shah, H. H., et al. Acute kidney injury in patients hospitalized with Covid-19. Kidney international , 98 (1), 209-218. https://doi.org/10.1016/j.kint.2020.05.006

Holshue, M. L., Chas DeBolt, C., Lofy, K, H., Wiesman, J., Bruce, H et al. (2020). First Case of 2019 Novel Coronavirus in the United States. The New England Journal of Medicine, 382 (10), 929-936. https://doi.org/10.1056/NEJMoa2001191

Kunutsor, S. K., \& Laukkanen, J. A. (2020). Renal complications in Covid-19: a systematic review and meta-analysis. Annals of medicine, 52(7), 345-353. https://doi.org/10.1080/07853890.2020.1790643

Martins, H; Brandão-Neto, R. A; Scalabrini-Neto, A, Velasco, I. Emergências Clínicas - Abordagem Prática. (9a ed.). Manole, 2014.

Moitinho, M. S., Belasco, A. G. S., Barbosa, D. A., \& Foncesa C. D. (2020). Acute Kidney Injury by SARS-CoV-2 virus in patients with Covid-19: an integrative review. Revista Brasileira de Enfermagem, 73 (2). https://doi.org/10.1590/0034-7167-2020-0354.

Nunes, L., \& Lima, T. (2021). Use of medicines for Covid-19 treatment in patients with loss of kidney function: a narrative review. Brazilian Journal of Nephrology, 43 (2), 2175-8239. https://doi.org/10.1590/2175-8239-JBN-2020-0105.

Pecly, I. M. D., Azevedo, R. B., Muxfeldt, E. S., Botelho, B. G., Albuquerque G. G., et al (2021). A review of Covid-19 and acute kidney injury: from pathophysiology to clinical results. Brazilian Journal of Nephrology., 43(4), 551-571. https://doi.org/10.1590/2175-8239-JBN-2020-0204.

Portolés, J., Marques, M., Sánchez, P. L., Valdenebro, M., Muñez, E et al. (2020). Doença renal crônica e lesão renal aguda no surto espanhol Covid-19, Transplante de diálise em nefrologia., 35 (8), 1353-1361. https://doi.org/10.1093/ndt/gfaa189

Queiroz A. G. S., Souza, R. Z., Sottocornola, S. F., Barbosa, S. J., Pinheiro, F. A., et al. (2020). Diagnósticos de enfermagem segundo a taxonomia da NANDA internacional para sistematização da assistência de enfermagem a Covid-19. J Health Biol Sci. 8(1):1-6. 10.12662/2317-3206jhbs.v8i1.3352.p1-6.2020

Scholz, J. R., Lopes, M. A. C. Q., Saraiva, J. F. K., \& Colombo, F. C. (2020). Covid-19, Sistema Renina-Angiotensina, Enzima Conversora da Angiotensina 2 e Nicotina: Qual é a Inter-relação?. Arq. Bras. Cardiol., 115 (4), 708-711. https://doi.org/10.36660/abc.20200653.

Sodré, F. L., Costa., J. C. B., \& Lima, J. C. C. (2007). Avaliação da função e da lesão renal: um desafio laboratorial. J. Bras. Patol. Med. Lab. 43 (5). https://doi.org/10.1590/S1676-24442007000500005

Sociedade Brasileira de Nefrologia. Insuficiência Renal Aguda. São Paulo: SN, 2007. https://www.sbn.org.br/fileadmin/user_upload/sbn/2020/01/0 4/Diretrizes_Insuficiencia_Renal_Aguda.pdf

Sosa-García, J. O., Gutiérrez-Villaseñor, A. O., García-Briones, A., Romero-González, J. P., Juárez-Hernández, E., \& González-Chon, O. (2020). Experience in the management of severe Covid-19 patients in an intensive care unit. Cirugia y cirujanos, 88(5), 569-575. https://doi.org/10.24875/CIRU.20000675

Suassuna, J. H. R., Lima, E. Q., Rocha, E., Castro, A., Burdmann, E. A., et al. Technical note and clinical instructions for Acute Kidney Injury (AKI) in patients with Covid-19: Brazilian Society of Nephrology and Brazilian Association of Intensive Care Medicine. Braz. J. Nephrol, 42(2 Supl. 1):22-31. https://doi.org/10.1590/2175-8239-JBN-2020-S107.

Tandukar, S., \& Palevsky, P. M. (2019). Continuous Renal Replacement Therapy: Who, When, Why, and How. Chest, 155(3), 626-638. https://doi.org/10.1016/j.chest.2018.09.004

Wang, X., Fang, X., Cai, Z., Wu, X., Gao, X., Min, J., \& Wang, F. (2020). Comorbid Chronic Diseases and Acute Organ Injuries Are Strongly Correlated with Disease Severity and Mortality among COVID-19 Patients: A Systemic Review and Meta-Analysis. Research (Washington, D.C.), 2402961. https://doi.org/10.34133/2020/2402961

Younes-Ibrahim, M. (2021). O rim: função, células e biomarcadores. Braz. J. Nephrol., 43 (1), 3-4. https://doi.org/10.1590/2175-8239- JBN-2020-0215 